\title{
Biomarkers in active surveillance
}

\author{
Stacy Loeb ${ }^{1,2,3}$, Jeffrey J. Tosoian ${ }^{4}$ \\ ${ }^{1}$ Department of Urology, ${ }^{2}$ Population Health, New York University, New York, NY, USA; ${ }^{3}$ The Manhattan VA, New York, NY, USA; ${ }^{4}$ The James \\ Buchanan Brady Urological Institute, Johns Hopkins School of Medicine, Baltimore, MD, USA \\ Contributions: (I) Conception and design: All authors; (II) Administrative support: All authors; (III) Provision of study material or patients: All authors; \\ (IV) Collection and assembly of data: All authors; (V) Data analysis and interpretation: All authors; (VI) Manuscript writing: All authors; (VII) Final \\ approval of manuscript: All authors. \\ Correspondence to: Stacy Loeb, MD, MSc. 550 1st Ave (VZ30, \#612), New York, NY 10016, USA. Email: stacyloeb@gmail.com.
}

\begin{abstract}
The use of active surveillance (AS) is increasing for favorable-risk prostate cancer. However, there remain challenges in patient selection for AS, due to the limitations of current clinical staging. In addition, monitoring protocols relying on serial biopsies is invasive and presents risks such as infection. For these reasons, there is substantial interest in identifying markers that can be used to improve AS selection and monitoring. In this article, we review the evidence on serum, urine and tissue markers in AS.
\end{abstract}

Keywords: Prostate cancer; active surveillance (AS); biomarkers; markers

Submitted Dec 11, 2017. Accepted for publication Dec 18, 2017.

doi: 10.21037/tau.2017.12.26

View this article at: http://dx.doi.org/10.21037/tau.2017.12.26

\section{Introduction}

Multiple guidelines now recommend active surveillance (AS) as the preferred approach for management of lowrisk prostate cancer $(1,2)$. Randomized trials have shown no difference in the risk of prostate cancer mortality between conservative management and radical treatment in these patients (3). Deferring initial therapy has the further advantage of allowing patients to preserve quality of life for additional years. However, uptake of AS has been highly variable (4), and it remains underutilized in the United States, due to multiple barriers at both the patient and physician level (5). One of the challenges is optimizing patient selection for AS, given the substantial heterogeneity within clinical risk categories and known risk of misclassification. Several markers are now available that can be used to refine risk classification and help with the initial decision to pursue AS.

Monitoring patients during AS remain another major challenge in contemporary practice. Changes in the total PSA level are not reliable predictors of underlying changes in biopsy features during the initial phase of AS (6). However, AS strategies that rely on frequent serial prostate biopsy are associated with added patient burden and risk of side effects. Of particular concern is the increase in infectious complications after biopsy, corresponding with a rising prevalence of antimicrobial resistance in the community (7). Several alternatives are available which have been associated with a lower risk of infection following prostate biopsy, such as use of targeted prophylaxis or using a transperineal approach (8). Nevertheless, biopsy remains an invasive and painful procedure, as well as a source of non-compliance for patients. This creates a need for other non-invasive markers that can be used to help with monitoring of patients during AS. The purpose of this article is to review the markers that can be used for selection and monitoring during AS.

\section{Serum markers}

Several studies have examined PSA kinetics during AS using biopsy endpoints, with primarily negative results. In the Johns Hopkins AS program, our group examined the relationship between PSA kinetics with the results of surveillance biopsy during a median follow-up of 2.9 years (6). PSADT was not significantly associated with adverse biopsy findings $(\mathrm{P}=0.83)$, and PSAV had a nonsignificant trend $(\mathrm{P}=0.06)$. Similar findings were reported in the Royal Marsden program, in which PSAV was significantly 
associated with histologic progression on univariable analysis but was not significant in the multivariable model $(\mathrm{P}=0.069)$ (9). At UCSF, a PSA doubling time within 3 years was not significantly associated with biopsy progression (OR, 1.4; 95\% CI, 0.6-3.4, P=0.46) (10). By contrast, in 120 men from Beth Israel they found that a PSAV $>0.11$ between the diagnostic biopsy and first rebiopsy was a significant independent predictor of reclassification. The authors created a risk score using PSAV along with family history and PSA density to further stratify the likelihood of biopsy reclassification (11). Notably, more recent studies have shown that PSA kinetics may be more useful for men who have already been stable on AS for several years. For example, Patel et al. reported that PSAV risk count (number of occasions that PSAV exceeded a threshold of $0.4 \mathrm{ng} / \mathrm{mL} /$ year) was a significant predictor of reclassification among men on AS for at least 2 years (12). Many programs currently use PSA kinetics as an indicator for further diagnostic testing such as MRI or biopsy, rather than as an independent trigger for intervention.

PSA density is another PSA-based parameter which has been studied extensively in AS, and is employed by several AS programs to determine upfront eligibility (13). This is based on studies showing that PSAD $>0.15$ is associated with an increased risk of biopsy reclassification and receipt of secondary treatment $(11,14,15)$. In the Prostate Cancer Research International: Active Surveillance (PRIAS) cohort, a PSAD $<0.2$ is one of the inclusion criteria for AS, while Johns Hopkins uses a lower threshold of PSAD $<0.15$ $(13,16)$. Reese et al. reported on men treated by prostatectomy who met some but not all of the Hopkins AS eligibility criteria (17). Overall, 1,205 (14.6\%) of the sample were excluded due to a PSAD between 0.15 and 0.2 , demonstrating that using a higher PSAD threshold would increase the number of men eligible for AS. However, they reported that men with a PSAD $0.15-0.18$ had a significantly higher risk of adverse features at prostatectomy compared to men with a PSAD $<0.15$.

Alternatively, the percent free PSA (\% fPSA) can be used along with the maximum percentage of core involvement to predict the results of the first surveillance re-biopsy and confirm eligibility for AS (18). Two new PSA-based blood tests incorporating free PSA are the Prostate Health Index (phi) and 4K score. Studies from the US and Asia have shown that baseline phi values predict reclassification at 1 year $(19,20)$. Longitudinal values of phi were also shown to predict progression during the course of AS (C-index 0.82). Although not yet studied in the setting of AS, phi density has demonstrated very good predictive value in the diagnostic setting and therefore could prove useful in AS (21). In the Canary Prostate Active Surveillance Study (PASS), the $4 \mathrm{~K}$ score at diagnosis was shown to improve accuracy for predicting reclassification at the first re-biopsy but not at subsequent AS biopsies (22).

\section{Urinary markers}

Several urinary markers have also been explored for use in prostate cancer detection and management. Data are limited in the realm of AS, but some groups have studied the cancer-specific messenger RNA known as prostate cancer antigen 3 (PCA3) and the TMPRSS2: ERG gene fusion.

In the setting of AS, PCA3 was initially explored in 294 men at Johns Hopkins with very low risk disease. Urinary PCA3 was measured at a median of 2.5 years following diagnosis, and patients were followed with yearly biopsy for a median of 3.7 years to determine reclassification. The data revealed an association between PCA3 and subsequent biopsy reclassification that did not meet conventional levels of statistical significance (mean value 50.8 and 60.0 in non-reclassified and reclassified, respectively; $\mathrm{P}=0.131$ ) (23). More recently, these authors performed a longitudinal analysis among AS patients with multiple PCA3 measures obtained over $\geq 3$ years of monitoring (24). Over median 6 years of follow-up, $28(11 \%)$ of 260 eligible men underwent grade reclassification. Men who underwent grade reclassification had a higher initial PCA3 (median 48.0 vs. 24.5, $\mathrm{P}=0.007$ ) and subsequent PCA3 (median 63.5 vs. 36.0, $\mathrm{P}=0.002$ ) than those who did not. Interestingly, the longitudinal change in PCA3 over time did not discriminate those who did and did not undergo grade reclassification (log-normalized rate $0.07 v s$. 0.06, $\mathrm{P}=0.53)$. Overall, these findings suggest that a single PCA3 level can help to predict pathological upgrading during AS, but obtaining multiple measurements over time does not appear to offer additional information.

The multi-institutional Canary PASS investigated both PCA3 and TMPRSS2:ERG in 387 men who were monitored with biopsy at 6 to 12 months from diagnosis, again at 24 months, and subsequently every 2 years (25). The authors observed a significant difference in PCA3 score based on follow-up biopsy results. Median PCA3 scores were 27 in men with a negative biopsy, 31 in those with Gleason score $\leq 6$ disease, and 48 in those with Gleason score $\geq 7(P=0.02)$. Median values of TMPRSS2:ERG were 5, 14, and 29, respectively $(\mathrm{P}=0.001)$. 
On ROC analysis for prediction of Gleason $\geq 7$ disease, the addition of these markers to PSA had an AUC of 0.70 versus 0.68 for PSA alone $(\mathrm{P}=0.08)$.

More recently, Fradet and colleagues explored the use of PCA3 in a unique cohort of AS patients with low-risk prostate cancer who were being treated with a 5 -alpha reductase inhibitor (5-ARI) (26). All men were treated with a 5 -ARI for at least 6 months (mean, 14.6 months; SD, 10) prior to urine specimen obtainment, which occurred after diagnosis but prior to the initial repeat biopsy. Over a mean follow-up of 45.6 months, 36 (40\%) of 90 men underwent grade reclassification to Gleason score $\geq 7$. Adjusting for age, prostate volume, and PSA, a PCA3 score $\geq 35$ was associated with a nearly four-fold increased hazard of grade reclassification (HR, 3.82; 95\% CI, 1.91-7.62). A significant relationship was also demonstrated when PCA3 was modeled as a continuous variable (HR, $1.13 ; 95 \% \mathrm{CI}$, 1.01-1.26; $\mathrm{P}=0.028)$.

Finally, Zhao et al. analyzed DNA methylation patterns of urinary sediment among 153 men with Gleason score 6 cancer monitored on AS (27). The authors considered eight candidate genes for which hypermethylation in radical prostatectomy specimens was associated with high grade cancer and adverse clinical outcomes. Over a median follow-up of 38 (range, 12-44) months, 34 (22\%) men were reclassified based on either Gleason score upgrading $(n=23)$, PSADT $<3$ years, or detecting a PI-RADS 4-5 lesion on MRI. Based on backward logistic regression, a four gene panel (APC, CRIP3, GSTP1, HOXD8) was associated with Gleason score upgrading during follow-up (OR, 2.927; 95\% CI, 1.264-6.779; $\mathrm{P}=0.012$ ). Validation in independent cohorts could better establish the utility of these and other urinary markers in the AS setting.

\section{Tissue markers}

Multiple tissue based markers are commercially available including the Oncotype DX Genomic Prostate Score (GPS) (28), Prolaris Cell Cycle Progression (CCP) score (29), GenomeDx Decipher score (30), and immunohistochemical (IHC) staining for inactivation of the PTEN gene (31). There is a growing body of evidence that tissue-based molecular testing provides prognostic information independent of clinical factors and may aid in decisionmaking after localized therapy $(32,33)$. Given the utility of these tests in predicting outcomes after treatment, questions have emerged as to whether they could prove useful in selection and monitoring during AS. Unfortunately, these tests have not been well-studied in a true AS population.

We have previously summarized the biologic basis of these tools and reviewed the evidence of their utility in risk stratification (34). Interim data specific to the AS setting have been sparse. In fact, recent reports of the genomic panels have been limited to demonstrating either clinical utility in decision-making (35), or incremental prognostic value among AS-eligible men who were managed definitively (36).

On the other hand, Lokman and colleagues retrospectively assessed PTEN IHC in 190 men managed in PRIAS (37). Tissue from the initial diagnostic biopsies demonstrated PTEN loss in 29 patients (15\%). Median cohort followup was 46.2 months, during which patients underwent between one and six surveillance biopsies. During followup, 106 men (52\%) discontinued AS. Seventy-two men (67.9\%) discontinued AS for protocol-based reasons (Grade group $>1,>2$ positive biopsy cores, PSA-DT $<3$ years, or clinical stage $>\mathrm{T} 2)$ and $34(32.1 \%)$ for other reasons. In multivariable Cox models, PTEN loss in the diagnostic biopsy was significantly associated with all measured outcomes, including grade group upgrading on rebiopsy (HR, 2.57; 95\% CI, 1.16-5.70; $\mathrm{P}=0.02$ ), treatment change (HR, 2.31; 95\% CI, 1.26-4.19; $\mathrm{P}=0.006$ ), and adverse pathology in men who underwent prostatectomy (HR, 4.75; 95\% CI, 1.84-12.23; $\mathrm{P}=0.001)$. The number of positive biopsy cores at diagnosis and number of rebiopsy sessions during follow-up were also associated with the study outcomes. These data suggest that the previously observed relationship of PTEN loss with adverse prostate cancer outcomes is consistent in the setting of AS.

\section{Future directions}

Very few markers have longitudinal results available among men being monitored during AS, so this is an important area for future research. Once more data become available on the use of markers through the course of AS, we must then determine how to integrate this information with other longitudinal data including the results of serial biopsies. Numerous nomograms are available in the screening context including new markers along with traditional risk factors to predict the risk of aggressive prostate cancer on biopsy. A few groups have recently designed nomograms to predict biopsy reclassification during AS, including the Canary Prostate Active Surveillance Study (including PSA along with clinico-pathologic features) (38) and Johns Hopkins (which includes PSA density along with other clinico-pathologic variables) (39). In the future as 
more data accrue, these AS calculators should be updated to incorporate new markers and imaging variables to facilitate the incorporation of multiple factors for clinical decision-making. In the future, customized decision support tools incorporating longitudinal patient data may facilitate a shift from protocolbased to more personalized AS monitoring (40).

\section{Acknowledgements}

Funding: This study was supported by The Edward Blank and Sharon Cosloy-Blank Family Foundation, The Gertrude and Louis Feil Family, the New York State Department of Health (\#DOH01-C30697GG-3450000), The Laura and Isaac Perlmutter Cancer Center at NYU Langone Medical Center (P30CA016087), and the National Institutes of Health (Award Number K07CA178258) to SL. The content is solely the responsibility of the authors and does not represent the official views of the NIH.

\section{Footnote}

Conflicts of Interest: S Loeb received honoraria for lectures from Astellas, MDxHealth and Boehringer Ingelheim, reimbursed travel from Sanofi and Astellas, and consulting for GE, GenomeDx Biosciences and Lilly (unrelated to the current manuscript). JJ Tosoian has no conflicts of interest to declare.

\section{References}

1. Chen RC, Rumble RB, Loblaw DA, et al. Active Surveillance for the Management of Localized Prostate Cancer (Cancer Care Ontario Guideline): American Society of Clinical Oncology Clinical Practice Guideline Endorsement. J Clin Oncol 2016;34:2182-90.

2. Clinically Localized Prostate Cancer: AUA/ASTRO/ SUO Guideline. Available online: http://www.auanet.org/ guidelines/clinically-localized-prostate-cancer-new-(aua/ astro/suo-guideline-2017)

3. Hamdy FC, Donovan JL, Lane JA, et al. 10-Year Outcomes after Monitoring, Surgery, or Radiotherapy for Localized Prostate Cancer. N Engl J Med 2016;375:1415-24.

4. Loeb S, Carter HB, Schwartz M, et al. Heterogeneity in active surveillance protocols worldwide. Rev Urol 2014;16:202-3.

5. Loeb S, Curnyn C, Fagerlin A, et al. A Qualitative Study on Decision-Making by Prostate Cancer Physicians during Active Surveillance. BJU Int 2017;120:32-9.

6. Ross AE, Loeb S, Landis P, et al. Prostate-specific antigen kinetics during follow-up are an unreliable trigger for intervention in a prostate cancer surveillance program. J Clin Oncol 2010;28:2810-6.

7. Loeb S, Vellekoop A, Ahmed HU, et al. Systematic Review of Complications of Prostate Biopsy. Eur Urol 2013;64:876-92.

8. Liss MA, Ehdaie B, Loeb S, et al. An Update of the American Urological Association White Paper on the Prevention and Treatment of the More Common Complications Related to Prostate Biopsy. J Urol 2017. [Epub ahead of print].

9. Venkitaraman R, Norman A, Woode-Amissah R, et al. Predictors of histological disease progression in untreated, localized prostate cancer. J Urol 2007;178:833-7.

10. Whitson JM, Porten SP, Hilton JF, et al. The relationship between prostate specific antigen change and biopsy progression in patients on active surveillance for prostate cancer. J Urol 2011;185:1656-60.

11. San Francisco IF, Werner L, Regan MM, et al. Risk stratification and validation of prostate specific antigen density as independent predictor of progression in men with low risk prostate cancer during active surveillance. J Urol 2011;185:471-6.

12. Patel HD, Feng $Z$, Landis $P$, et al. Prostate specific antigen velocity risk count predicts biopsy reclassification for men with very low risk prostate cancer. J Urol 2014;191:629-37.

13. Tosoian JJ, Mamawala M, Epstein JI, et al. Intermediate and Longer-Term Outcomes From a Prospective ActiveSurveillance Program for Favorable-Risk Prostate Cancer. J Clin Oncol 2015;33:3379-85.

14. Dall'Era MA, Konety BR, Cowan JE, et al. Active surveillance for the management of prostate cancer in a contemporary cohort. Cancer 2008;112:2664-70.

15. Barayan GA, Brimo F, Begin LR, et al. Factors influencing disease progression of prostate cancer under active surveillance: a McGill University Health Center cohort. BJU Int 2014;114:E99-E104.

16. Bul M, Zhu X, Valdagni R, et al. Active surveillance for low-risk prostate cancer worldwide: the PRIAS study. Eur Urol 2013;63:597-603.

17. Reese AC, Landis P, Han M, et al. Expanded criteria to identify men eligible for active surveillance of low risk prostate cancer at Johns Hopkins: a preliminary analysis. J Urol 2013;190:2033-8.

18. Tseng KS, Landis P, Epstein JI, et al. Risk Stratification of Men Choosing Surveillance for Low Risk Prostate Cancer. J Urol 2010;183:1779-85.

19. Tosoian JJ, Loeb S, Feng Z, et al. Association of [-2] proPSA with Biopsy Reclassification During Active 
Surveillance for Prostate Cancer. J Urol 2012;188:1131-6.

20. Hirama H, Sugimoto $M$, Ito K, et al. The impact of baseline [-2]proPSA-related indices on the prediction of pathological reclassification at 1 year during active surveillance for low-risk prostate cancer: the Japanese multicenter study cohort. J Cancer Res Clin Oncol 2014;140:257-63.

21. Tosoian JJ, Druskin SC, Andreas D, et al. Prostate Health Index density improves detection of clinically significant prostate cancer. BJU Int 2017;120:793-8.

22. Lin DW, Newcomb LF, Brown MD, et al. Evaluating the Four Kallikrein Panel of the 4Kscore for Prediction of High-grade Prostate Cancer in Men in the Canary Prostate Active Surveillance Study. Eur Urol 2017;72:448-54.

23. Tosoian JJ, Loeb S, Kettermann A, et al. Accuracy of PCA3 measurement in predicting short-term biopsy progression in an active surveillance program. J Urol 2010;183:534-8.

24. Tosoian JJ, Patel HD, Mamawala M, et al. Longitudinal assessment of urinary PCA3 for predicting prostate cancer grade reclassification in favorable-risk men during active surveillance. Prostate Cancer Prostatic Dis 2017;20:339-42.

25. Lin DW, Newcomb LF, Brown EC, et al. Urinary TMPRSS2:ERG and PCA3 in an active surveillance cohort: results from a baseline analysis in the Canary Prostate Active Surveillance Study. Clin Cancer Res 2013:19:2442-50.

26. Fradet V, Toren P, Nguile-Makao M, et al. Prognostic value of urinary prostate cancer antigen 3 (PCA3) during active surveillance of patients with low-risk prostate cancer receiving 5alpha-reductase inhibitors. BJU Int 2017. [Epub ahead of print].

27. Zhao F, Olkhov-Mitsel E, van der Kwast T, et al. Urinary DNA Methylation Biomarkers for Noninvasive Prediction of Aggressive Disease in Patients with Prostate Cancer on Active Surveillance. J Urol 2017;197:335-41.

28. Knezevic D, Goddard AD, Natraj N, et al. Analytical validation of the Oncotype DX prostate cancer assay a clinical RT-PCR assay optimized for prostate needle biopsies. BMC Genomics 2013;14:690.

29. Cuzick J, Swanson GP, Fisher G, et al. Prognostic value of an RNA expression signature derived from cell cycle proliferation genes in patients with prostate cancer: a retrospective study. Lancet Oncol 2011;12:245-55.

30. Erho N, Crisan A, Vergara IA, et al. Discovery and validation of a prostate cancer genomic classifier that predicts early metastasis following radical prostatectomy.
PLoS One 2013;8:e66855.

31. Lotan TL, Gurel B, Sutcliffe S, et al. PTEN protein loss by immunostaining: analytic validation and prognostic indicator for a high risk surgical cohort of prostate cancer patients. Clin Cancer Res 2011;17:6563-73.

32. Nguyen PL, Haddad Z, Ross AE, et al. Ability of a Genomic Classifier to Predict Metastasis and Prostate Cancer-specific Mortality after Radiation or Surgery based on Needle Biopsy Specimens. Eur Urol 2017;72:845-52.

33. Nguyen PL, Shin H, Yousefi K, et al. Impact of a Genomic Classifier of Metastatic Risk on Postprostatectomy Treatment Recommendations by Radiation Oncologists and Urologists. Urology 2015;86:35-40.

34. Tosoian JJ, Loeb S, Epstein JI, et al. Active Surveillance of Prostate Cancer: Use, Outcomes, Imaging, and Diagnostic Tools. Am Soc Clin Oncol Educ Book 2016;35:e235-45.

35. Eure G, Germany R, Given R, et al. Use of a 17-Gene Prognostic Assay in Contemporary Urologic Practice: Results of an Interim Analysis in an Observational Cohort. Urology 2017;107:67-75.

36. Tosoian JJ, Chappidi MR, Bishoff JT, et al. Prognostic utility of biopsy-derived cell cycle progression score in patients with National Comprehensive Cancer Network low-risk prostate cancer undergoing radical prostatectomy: implications for treatment guidance. BJU Int 2017;120:808-14.

37. Lokman U, Erickson AM, Vasarainen H, et al. PTEN Loss but Not ERG Expression in Diagnostic Biopsies Is Associated with Increased Risk of Progression and Adverse Surgical Findings in Men with Prostate Cancer on Active Surveillance. Eur Urol Focus 2017. [Epub ahead of print].

38. Ankerst DP, Xia J, Thompson IM Jr, et al. Precision Medicine in Active Surveillance for Prostate Cancer: Development of the Canary-Early Detection Research Network Active Surveillance Biopsy Risk Calculator. Eur Urol 2015;68:1083-8.

39. Mamawala MM, Rao K, Landis P, et al. Risk prediction tool for grade re-classification in men with favourable-risk prostate cancer on active surveillance. BJU Int 2017;120:25-31.

40. Loeb S. Shift from protocol-based to personalized medicine in active surveillance: beginning of a new era. BJU Int 2017;120:3-4.

Cite this article as: Loeb S, Tosoian JJ. Biomarkers in active surveillance. Transl Androl Urol 2018;7(1):155-159. doi: $10.21037 /$ tau.2017.12.26 